Marquette University

e-Publications@Marquette

College of Nursing Faculty Research and

Publications

Nursing, College of

$1-1-2017$

\title{
Addressing Traumatic Stress in the Acute Traumatically Injured Patient
}

Claire Frank

University of Wisconsin-University Hospital

Kathryn Schroeter

Marquette University, kathryn.schroeter@marquette.edu

Chris Shaw

Marquette University

Accepted version. "Addressing Traumatic Stress in the Acute Traumatically Injured Patient," in Journal of Trauma Nursing, Vol. 24, No. 2 (2017): 78-84. DOI. (C) 2017 Lippincott Williams \& Wilkins, Inc. Used with permission. 


\title{
Marquette University
}

\section{e-Publications@Marquette}

\section{Nursing Faculty Research and Publications/College of Nursing}

This paper is NOT THE PUBLISHED VERSION; but the author's final, peer-reviewed manuscript. The published version may be accessed by following the link in the citation below.

Journal of Trauma Nursing, Vol. 24, No. 2 (March/April 2017): 78-84. DOI. This article is (C) Society of Trauma Nurses and permission has been granted for this version to appear in ePublications@Marquette. Society of Trauma Nurses does not grant permission for this article to be further copied/distributed or hosted elsewhere without the express permission from Society of Trauma Nurses.

\section{Addressing Traumatic Stress in the Acute Traumatically Injured Patient}

\author{
Claire A. Frank
}

University of Wisconsin-University Hospital, Madison

\section{Kathryn Schroeter}

Marquette University, Milwaukee, Wisconsin

\section{Chris Shaw}

Marquette University, Milwaukee, Wisconsin

\section{Abstract}

Psychological injuries after an acute traumatic event are commonly overlooked. Currently within United States, there is no consistently utilized screening process that addresses traumatic stress within the acute trauma population. Roy's Adaptation Model guided this project, focusing on the idea that bedside nurses are at the frontline of providing early identification through nursing assessment. The purpose of this pilot study was to evaluate whether the implementation of the Primary Care-Posttraumatic Stress Disorder (PC-PTSD) screening tool by bedside nurses would result in identifying more patients at risk for traumatic stress after an acute trauma as compared with the use of no screening method. This descriptive survey study took place over a 6-week period at a Midwest Level 1 trauma hospital. The results revealed that the tool did not increase the 
number of health psychology consults when compared with the same 6-week period a year prior when no tool was used. Nonetheless, use of the PC-PTSD tool did trigger $28 \%$ of the patients to receive a health psychology consult. Forty-five percent of patients who received a health psychology consult were recommended outpatient therapy. Utilization of this tool by bedside nurses did not adversely increase a number of inappropriate health psychology consults. These results agree with the literature and further suggest that there are a clinically significant number of acute trauma patients who are at risk for traumatic stress. With this screening tool, nurses accurately assessed patients and connected them with timely psychological treatment.

Traumatic injuries are a common occurrence within the United States, as each year it is estimated that a traumatic event accounts for 2.3 million hospital admissions across the United States (National Trauma Institute [NTI], 2014). Internationally, tens of millions of people who encounter a traumatic event suffer nonthreatening physical injuries that require ongoing medical treatment (World Health Organization [WHO], 2014). A majority of the medical treatment after a traumatic event is focused on caring for the physical needs by immediately providing airway support, hemodynamic stabilization, fixation of fractured bones, and control of other potential life-threatening risks. This focus will always remain a top priority for trauma patients, yet what are commonly overlooked are the emotional or psychological injuries that can ensue after a traumatizing event.

In the most recent edition from the American College of Surgeons "Resources for Optimal Care of the Injured Patient" (2014), it was recognized that disregarding the psychological symptoms after a traumatic event can leave patients vulnerable for the development of maladaptive behaviors. These behaviors can lead to detrimental symptoms that limit a patient's return to full life activity and function. One such maladaptive psychological disorder recognized by the American Psychological Association (2000), Diagnostic and Statistical Manual of Mental Disorders (DSM-V), and can be found in the acute trauma population is acute stress disorder (ASD). Acute stress disorder is also closely linked to the more chronic diagnosis of posttraumatic stress disorder (PTSD). What is unique within the definition of ASD is that these pathologic symptoms can be present as soon as 2 days after a traumatic event (American Psychiatric Association [APA], 2000). Any symptoms that persist longer than 30 days are then considered within the diagnosis of PTSD.

\section{BACKGROUND AND LITERATURE}

The prevalence of ASD following a traumatic event has been found to range from $5 \%$ to $25 \%$ depending on the type of trauma that the individual encountered (Harvey \& Bryant, 1998; Harvey \& Bryant, 1999; Holeva, Tarrier, \& Wells, 2001). Development of ASD can also increase the risk of developing subsequent PTSD (Harvey \& Bryant, 1998; Holeva et al., 2001). Harvey and Bryant (1998) found that $13 \%$ of motor vehicle crash (MVC) survivors were diagnosed with ASD. In follow-up visits with the same patients, $78 \%$ of the ASD-positive participants then met the criteria for PTSD. A large prospective cohort study assessed PTSD rates after any type of acute traumatic event that led to an injury (Zatzick et al., 2007). Zatzick et al. (2007) found that $23 \%$ of injury survivors had symptoms consistent with a diagnosis of PTSD 12 months after their acute inpatient hospitalization. Within this literature, it is evident that individuals who are involved in a traumatic experience are at risk for the development of ASD and subsequent PTSD.

To prevent negative traumatic stress responses, it is necessary to address it. Early identification of traumatic stress offers health care providers the opportunity to implement early interventions that prevent at-risk individuals from progression to worse outcomes (Bryant, 2006; Zatzick et al., 2011). Various international guidelines highlight the importance of this early identification (Australian Centre for Posttraumatic Mental Health [ACPMH], 2013; Department of Veterans Affairs/Department of Defense [VA/DoD], 2010; National Institute for Clinical Excellence [NICE], 2005). The U.S. Department of Veteran Affairs (2010) identified that those at higher risk for traumatic stress should be recognized by using pretraumatic risk factors, pretraumatic factors, and posttraumatic factors. These factors include lifestyle, preinjury stress, physical injuries, role failure, and social support ( $\mathrm{VA} / \mathrm{DoD}, 2010)$. Identification of these risk factors can assist in focusing on those higher risk patients; yet, this assessment may not be as applicable in the acute care setting. Many of the 
current health care systems rely on clinical providers, such as nurses, to identify these risk factors as an aspect of usual care (Bertelson, Brasel, \& deRoon-Cassini, 2011). It has been found that this usual care is commonly unsuccessful, as a majority of nurses caring for these patients feel inadequately trained to recognize detrimental symptoms and seek appropriate referral (Alexander \& Atcheson, 1998; Bertelson et al., 2011; Lee

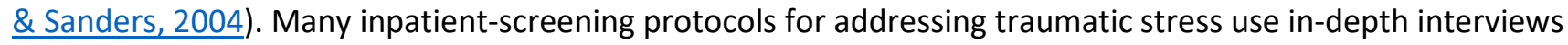
that can be time-consuming and lengthy, which is not always feasible in this acute patient population (Hanley, deRoon-Cassini, \& Brasel, 2013; Lee, Brasel, \& Lee, 2004). Within the inpatient setting, there has been a call for shorter screening tools to help clinical providers, such as nurses, recognize symptoms and then initiate follow-up with the use of diagnostic screening performed by a certified psychiatric provider (Bertelson et al., 2011).

One tool that has been proven to have superior screening efficiency is the Primary Care-Posttraumatic Stress Disorder (PC-PTSD) tool (Prins et al., 2003). What is appealing about this tool is that it consists of only four questions and takes less than a minute to administer. Each of the four questions specifically addresses the corresponding symptoms that are present in the diagnostic criteria of PTSD, but these symptoms also fall within the DSM-V diagnostic criteria of ASD. One study performed by Hanley et al. (2013) utilized the PC-PTSD tool in the acute trauma care setting finding that the PC-PTSD tool retained a high specificity in identifying those at risk for ASD at $93.4 \%$. This tool is also one that is utilized for screening only and is not to serve as a diagnostic measure.

The Roy Adaptation Model (RAM) was utilized to guide the implementation of a study that assesses for traumatic stress in the acutely injured patient. The RAM is a theoretical nursing model that presents the idea that human adaptation is needed to maintain integrity in one's life (Roy, 2009). Use of this nursing theory can help conceptualize the adaptive responses that may follow after a traumatic event. Roy (2009) discussed that the only way to know how an individual is adapting to a situation is to observe his or her output behavior. Maladaptive symptoms associated with traumatic stress can lead to harmful behaviors that limit an individual's quality of life. Influencing these output behaviors are the coping mechanisms or adaptation modes that an individual possesses prior to his or her health situation (Roy \& Andrew, 1999). Roy considers the adaptation modes as a framework for nursing assessment and, therefore, is a major focus of this study. The use of nursing assessment plays a key role in preventing traumatic stress by providing early identification and early intervention.

\section{PURPOSE}

The purpose of this study is to assess the underestimation of traumatic stress within the acute trauma patient population by cultivating a standardized method of efficiently screening trauma patients who are at risk of developing negative traumatic stress symptoms after their traumatic event. It is hypothesized that the utilization of the PC-PTSD screening tool by bedside nurses will help identify more patients who are at risk of maladaptive traumatic stress. In addition, use of the screening tool by bedside nurses will trigger more readily available inpatient resources for these patients before they discharge.

\section{METHODS}

This study was a pre-/postdescriptive survey design. The study took place at a Level I Midwest trauma hospital on a trauma/surgical intermediate and general care inpatient unit. Those recruited for this study were any trauma patients who were admitted to this unit under the Trauma/Surgical team and had a length of stay (LOS) greater than $48 \mathrm{hr}$. Those excluded were any patients younger than 18 years or those patients who had a Glasgow Coma Scale score of less than 15. The intervention phase of this study took place over the designated 6-week period of February 1, 2016, to March 12, 2016. Comparison demographic data were obtained from the PC-PTSD screening tools and from the University of Wisconsin Trauma Registry.

Retrospective chart review was used to obtain the number of health psychology consults placed in a 6-week period in year 2015 and the number of patients who were recommended outpatient services by the health psychology consult team within the time period in year 2016. Protection for human subjects was met by 
obtaining institutional review board (IRB) approval through both the Level 1 hospital in which this study was performed and through a university IRB board.

The instrument used to screen trauma patients in this study was the PC-PTSD tool. The PC-PTSD tool was developed by government employees and is considered to be public domain (VA/DoD, 2016). It is not copyrighted and so no formal permission was needed and no cost was applied for the use of the tool. Each of the four questions within the PC-PTSD tool corresponds to symptoms that can be present in ASD or PTSD. A threshold score of answering "yes" to three or more of the four questions on the PC-PTSD tool has a high indication of identifying individuals at risk of developing subsequent PTSD and is, therefore, considered a positive result (Prins et al., 2003). This tool has been shown to have sensitivity ranging from $78 \%$ to $85 \%$ and specificity ranging from $78 \%$ to $82 \%$ (Freedy et al., 2010; Reese et al., 2012) Test-retest reliability for the PCPTSD scale has been found to be 0.83 (Spoont et al., 2013).

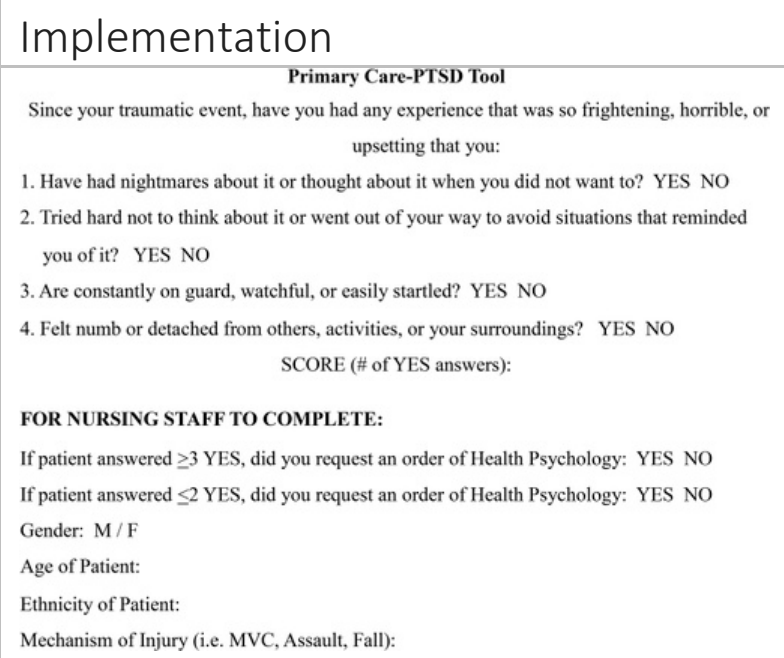

Figure 1. Primary Care-PTSD screening form (Prins et al., 2003). This adapted figure illustrates the tool that nurses used to screen inpatient trauma patients.

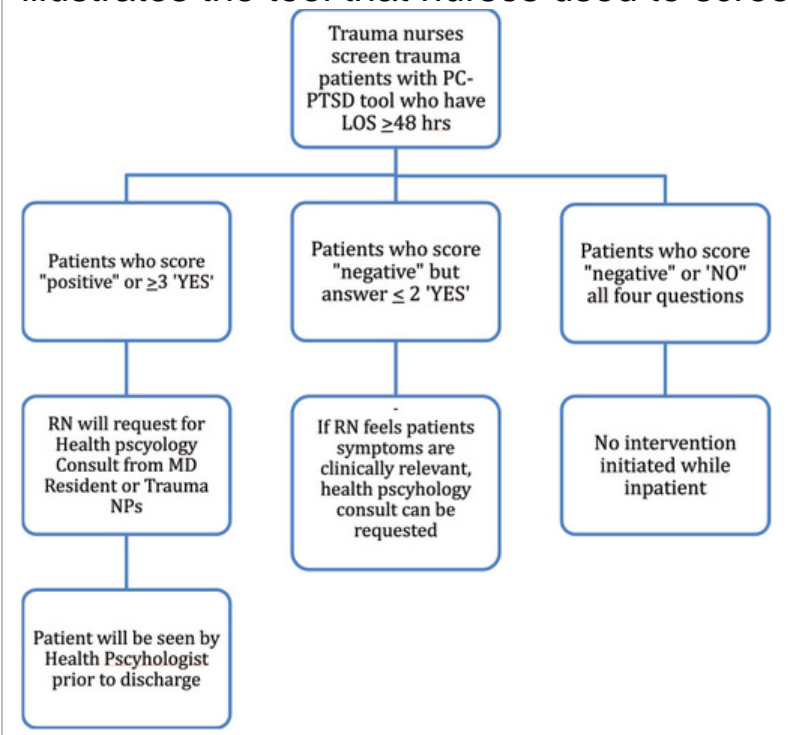

Figure 2. Implementation algorithm. This figure illustrates the algorithm of interventions that the trauma nurses followed in screening the inpatient trauma patients. LOS = length of stay; PC-PTSD = Primary CarePosttraumatic Stress Disorder.

The PC-PTSD screening tool (Figure 1) was administered by the trauma nurses on the pilot unit to any patient who had an LOS to or longer than $48 \mathrm{hr}$. The nurses then followed the implementation algorithm to assist in identifying subsequent interventions (Figure 2). Demographic information in regard to patient's age, gender, ethnicity, and mechanism of injuries was provided by the nurse at the bottom of the screening 
form. Completed forms were then returned to the project leader. The number of health psychology orders and recommended outpatient referrals were provided to the project leader by the lead health psychologist. After the 6 weeks of implementation, the following outcome measures were analyzed with the use of descriptive statistics:

* Total number of patients screened and percentage of those who had a score of 3 or greater.

* Number and percentage of patients who received a health psychology consult with implementation of the PC-PTSD tool, looking both at those who had scored 3 or greater and those with scores of 2 or less.

* Number and percentage of health psychology-consulted patients who were recommended to receive outpatient psychological care.

* Number and percentage of trauma patients who received a health psychology consult during the same 6-week time period 1 year prior when no tool was in place.

\section{RESULTS}

During the 6-week intervention period, 45 patients were admitted to the pilot unit for an LOS longer than 48 hr. Of these 45 patients, 40 had completed the PC-PTSD tool with their day shift nurse, yielding an $89 \%$ response rate. All PC-PTSD surveys with completed answers to the PC-PTSD tool were included in the data analysis. Only one survey did not have completed demographic information but did include the patient's answers to the PC-PTSD survey.

TABLE 1 Intervention Group Demographics

\begin{tabular}{|l|l|l|l|l|l|l|}
\hline & Male, $\boldsymbol{n}$ & Male, $\%$ & Female, $\boldsymbol{n}$ & Female, \% & Total, $\boldsymbol{n}$ & Total, \% \\
\hline Overall & 23 & & 16 & & 39 & \\
\hline Age, years & & & & & & \\
\hline $18-30$ & 5 & 62.5 & 3 & 37.5 & 8 & 21 \\
\hline $31-40$ & 4 & 100 & 0 & 0 & 4 & 10 \\
\hline $41-50$ & 5 & 71.5 & 2 & 28.5 & 7 & 18 \\
\hline $51-60$ & 5 & 62.5 & 3 & 37.5 & 8 & 21 \\
\hline $61-70$ & 2 & 50 & 2 & 50 & 4 & 10 \\
\hline $71-80$ & 2 & 50 & 2 & 50 & 4 & 10 \\
\hline $81+$ & 0 & 100 & 4 & 100 & 4 & 10 \\
\hline Mechanism of injury & & & & & & \\
\hline MVC & 9 & 45 & 11 & 55 & 20 & 51 \\
\hline Fall & 3 & 37.5 & 5 & 62.5 & 8 & 21 \\
\hline Assault & 2 & 100 & 0 & 0 & 2 & 5 \\
\hline Crush injury & 4 & 100 & 0 & 0 & 4 & 10 \\
\hline GSW & 1 & 100 & 0 & 0 & 1 & 3 \\
\hline Other & 4 & 100 & 0 & 0 & 4 & 10 \\
\hline Ethnicity & & & & & & \\
\hline Caucasian & 21 & 60 & 14 & 40 & 35 & 90 \\
\hline Pacific Islander & 1 & 100 & 0 & 0 & 1 & 3 \\
\hline Hispanic & 1 & 33 & 2 & 67 & 3 & 7 \\
\hline
\end{tabular}

Note. GSW = gun shot wound; $M V C=$ motor vehicle crash.

\section{TABLE 2 Pre-/Postimplementation Comparison Demographics}

\begin{tabular}{|c|c|c|}
\hline & February 1, 2015, to March 12, 2015 & 2016 \\
\hline Total Admitted Patients on Pilot Unit & $\mathrm{n}=38$ & $\mathrm{n}=45$ \\
\hline
\end{tabular}




\begin{tabular}{|l|c|c|}
\hline Average LOS & 6 & 6.6 \\
\hline Gender & 19 & 17 \\
\hline Female & 19 & 28 \\
\hline Male & & \\
\hline Age, years & 6 & 7 \\
\hline $18-25$ & 5 & 3 \\
\hline $26-35$ & 2 & 3 \\
\hline $36-45$ & 4 & 11 \\
\hline $46-55$ & 8 & 5 \\
\hline $56-65$ & 13 & 16 \\
\hline$>66$ & & 0 \\
\hline Race & 1 & 2 \\
\hline Asian & 0 & 4 \\
\hline Hispanic & 5 & 39 \\
\hline Black & 32 & 19 \\
\hline Caucasian & & 17 \\
\hline Most common mechanism of injury & 19 & 12 \\
\hline Fall & 12 & \\
\hline MVC & 19 & \\
\hline
\end{tabular}

Note. All demographics provided by UW Trauma Registry. LOS = length of stay; MVC = motor vehicle crash.

Demographics of the 39 patients screened during this period are summarized in Table 1 and pre/postintervention demographics are summarized in Table 2. Of these patients, 23 were male and 16 were female, with a mean age of 51.3 years for all patients. The sample consisted of 31 Caucasians (90\%), followed by three Hispanics and one Pacific Islander. Fifty-one percent of the intervention group was involved in MVC, with the second most common injury being falls (21\%).

Of the forty patients administered the screening tool during the implementation phase, five patients (12\%) scored positive, which was indicated by answering "yes" to three or more of the PC-PTSD tool questions. All five of these patients received a health psychology consult. Thirty-five patients $(88 \%)$ scored negative on the PC-PTSD, yet six (17\%) of these patients still received a health psychology order. The request for these orders was indicated by nursing judgment. In total of all screened patients, $11(28 \%)$ of the 40 patients screened received a health psychology consult with the use of the PC-PTSD tool.

During the preintervention comparison time frame of February 1, 2015, to March 12, 2015, there was a total of 16 health psychology consults placed among the trauma inpatient service when no tool was used. Between this same time period in year 2016 when the implementation phase took place, there was also 16 health psychology consults placed on the trauma inpatient service. Use of the PC-PTSD tool on the pilot unit triggered 11 of the 16 health psychology consults during the intervention phase in year 2016. Six total hospital-wide trauma patients who were consulted by the health psychology team were recommended to receive ongoing outpatient psychology services after discharge. Five of these patients were patients identified for a health psychology consult by the use of the PC-PTSD tool on the pilot unit.

\section{DISCUSSION}

It has been established in the literature that early identification and treatment of traumatic stress is vital in helping trauma patients optimize their recovery (Bryant, 2006). Studies that have explored the use of standardized tools for identification of early maladaptive stress recognize that "many trauma programs do not routinely screen for PTSD after a traumatic injury" (Hanley et al., 2013, p. 723). In addition, commonly used screening tools have failed to succeed in identifying at-risk patients within the acute trauma population. The primary purpose of this study was to explore the use of a short standardized method (PC-PTSD tool) that 
screened for traumatic stress disorders and to observe whether the use of this standardized tool would increase the number of acute trauma patients who receive appropriate and prompt psychological care. The results of this study found that this was not necessarily the case as there was the same number of health psychology consult orders placed between the two comparison time frames. Although this may seem to not reflect the original hypothesis, it does not negate the indication that utilization of this screening tool failed or that addressing traumatic stress is irrelevant. It is not uncommon for trauma patients to display symptoms of distress after a traumatic event, as it has been found that many learn to adapt within 3-6 weeks (Bryant, 2006; Warren, Stucky, \& Sherman, 2014). Although there was the same amount of health psychology consults, it is encouraging to find that the use of the tool did not adversely increase the amount of inappropriate consults. This is also considered when observing the number of patients who were recommended continued outpatient care. Of the total 11 patients who were consulted to see health psychology, five (45\%) of them were recommended outpatient services. This outcome can be seen as clinically significant in that those who were screened and seen by health psychology were deemed as higher risk for traumatic stress disorders by needing this additional follow-up care.

The percentage of patients who were consulted with health psychology for being at risk for traumatic stress disorder was also similar to other studies that performed screening for maladaptive traumatic stress (Bell, Sobolev, Anderson, Hewko, \& Simons, 2014; Bertelson et al., 2011; Reese et al., 2012; Zatzick et al., 2007). In this study, $28 \%$ of the patients received a health psychology consult with the use of the PC-PTSD tool. In addition, the response rate was very high for this study, suggesting that use of this short screening tool was overall easy to implement among the nursing staff. The five patients who were missed can be attributed to being either missed by nursing staff or not falling within the inclusion criteria of this study.

Nurses play an essential role in utilizing their critical thinking intuition to assess patients for psychological issues and are in a position to make the biggest impact on providing early identification to advocate for proper treatment for patients. Required use of a standardized tool can heighten a nurse's awareness to assess his or her patients for psychological issues. Continued nursing education did play large part of this study as it took time to enable nurses in feeling comfortable in discussing the issues of traumatic stress with their patients. These conversations help strengthen the bond only between the patient and his or her nurse. Although $88 \%$ of patients were screened "negative" on the PC-PTSD tool, an improved sensitivity to the patient's psychological health can increase the requests for appropriate referral. The use of this tool by bedside nurses helped identify at least five patients who were recommended continued outpatient psychology consultation. This result indicated that their symptoms were severe enough to interfere with return-to-life function and needed continued follow-up (Warren et al., 2014).

\section{LIMITATIONS}

There were several limitations within this pilot study. First, this study had a limited time frame for implementation of utilizing the PC-PTSD tool providing a smaller sample size. A smaller sample size could also account for the fact that this was a new tool introduced to the nursing staff on the designated inpatient unit. Continued education to the nurses was provided to help initiate screening the trauma patients, as $12 \%$ of the patients were missed. In addition, this was a new tool utilized by the bedside nurse, and there could be variability in how the tool was implemented. Nurses could have asked the patient the questions or had the patient fill out the questions himself or herself. This variability could introduce some bias as patients who were asked the questions may have been more hesitant to answer truthfully given possible stigma related to psychological disorders.

Another limitation is missing the number of patients who were seen by health psychology within the 2015 group and who were then recommended to receive outpatient care. This comparison would have been beneficial to know so that analysis of the PC-PTSD tool could be obtained. Having these data would have provided a better understanding of the sensitivity of the PC-PTSD tool compared to a time when no tool was utilized.

Finally, the PC-PTSD tool is a validated screening tool that has continued to show continued reliability and validity, but many of these studies take place in the primary care setting. It should be again noted that this 
tool was implemented in an acute care setting, which may not leave enough time for symptoms of traumatic stress to fully develop. Regardless, this tool was utilized as a screening tool and not for a diagnostic purpose.

\section{CONCLUSION}

The results of this pilot study did not support the original hypothesis that with the implementation of a standardized screening tool there would be more identification of patients who are at risk for maladaptive stress. Regardless, there were clinically significant results finding that use of the PC-PTSD assisted in identifying patients who need health psychology, and many of them were recommended to receive outpatient health psychology. The percentage of patients deemed at risk was also similar to other studies that address the prevalence of traumatic stress in the trauma population (Bell et al., 2014; Bertelson et al., 2011; Reese et al., 2012; Zatzick et al., 2007). These findings can positively affect the patient in the primary care setting as well. Avoidance of addressing this issue only predisposed these patients with maladaptive behaviors when they return to home. In addition, insurance coverage and waiting lists for outpatient psychological care can be a barrier for many patients. Providing a starting point of interventions within the inpatient setting assists only in the development of better coping mechanisms for patients when they return to their daily lives.

Facilitating the use of evidence-based care that supports interdisciplinary teamwork to identify trauma patients with traumatic stress assists in preventing unnecessary consequences of psychological issues. Practicing holistic care for patients is of utmost importance in providing the best care for these patients and helping them return to a high quality of life. Providing patients this style of care could also improve their inpatient experience after a traumatizing event. Further research could explore the use of the PC-PTSD tool for longer periods of time with a larger number of patients in the acute care setting or within outpatient trauma clinics. In addition, providing patients with suggestive resources, such as online options, upon discharge may also be an intervention that could be further examined to help patient's self-identify and reach out for supportive therapy.

\section{KEY POINTS}

* Nurses have an essential role in utilizing their critical thinking intuition to assess patients for psychological issues and are in a position to make the biggest impact on providing early identification and advocating proper treatment for these patients.

* Implementation of the PC-PTSD tool can be used as a standardized screening tool to assess acute traumatically injured patients to initiate early psychological resources upon discharge.

* Further research can explore the use the PC-PTSD tool within the acute trauma population or within trauma outpatient clinics.

\section{Acknowledgments}

The authors extend their gratitude to the hard work of the University of Wisconsin Hospital Trauma Level 1 trauma team, nurses on F4/4, UW Health-Health Psychology department, and the University of Wisconsin Hospital and Clinics Trauma Registry. The authors also thank the kind mentors at Marquette University.

\section{REFERENCES}

Alexander D. A., Atcheson S. F. (1998). Psychiatric aspects of trauma care survey of nurses and doctors. Psychiatric Bulletin, 22, 132-136.

American College of Surgeons. (2014). Rehabilitation. In Rotondo M. F., Cribari C., Smith R. S. (Eds.), Resources for optimal care of the injured patient (5th ed., pp. 89-93). Chicago, IL: American College of Surgeons 
American Psychiatric Association (APA). (2000). The diagnostic and statistical manual of mental disorders (4th ed.). Washington, DC: American Psychiatric Association.

Australian Centre for Posttraumatic Mental Health (ACPMH). (2013). Australian guidelines for the treatment of acute stress disorder and post traumatic stress diso rder. Melbourne, Victoria, Australia: Australian Centre for Posttraumatic Mental Health.

Bell N., Sobolev B., Anderson S., Hewko R., Simons R. K. (2014). Routine verses ad hoc screening for acute stress following injury: Who would benefit and what are the opportunities for prevention. Journal of Trauma Management and Outcomes, 8(5), 1-7. doi:10.1186/1752-2897-8-5

Bertelson A., Brasel K. J., deRoon-Cassini T. A. (2011). Implementing a posttraumatic stress and functional outcome screening process for trauma patients as a level 1 adult trauma center. Journal of Trauma Nursing, 18(1), 5-8. doi:10.1097/JTN.0b013e31820e3e40

Bryant R. A. (2006). Acute stress disorder. Psychiatry, 5(7), 238-239. doi:10.1053/j.mppsy.2006.04.003 Department of Veterans Affairs/Department of Defense (VA/DoD). (2010). Clinical practice guideline for the management of posttraumatic stress, version 1.0. Washington, DC: Veterans.

Department of Veterans Affairs/Department of Defense (VA/DoD). (2016). PTSD: National center for PTSD. Retrieved from: http://http://www.ptsd.va.gov/professional/assessment/screens/pc-ptsd.asp

Freedy J. R., Steenkamp M. M., Magruder K. M., Yeager D. E., Zoller J. S., Hueston W. J., Carek P. J. (2010). Post-traumatic stress disorder screening test performance in civilian primary care. Journal of Family Practice, 27, 615-624. doi:10.1093/fampra/cmq049

Hanley J., deRoon-Cassini T., Brasel K. (2013). Efficiency of a four-item posttraumatic stress disorder screen in trauma patients. Journal of Trauma and Acute Care Surgery, 75(4), 722-727.

doi:10.1097/TA.0b013e3182a53a5f

Harvey A. G., Bryant R. A. (1998). Acute stress disorder across trauma populations. Journal of Nervous and Mental Disease, 183, 443-446.

Harvey A. G., Bryant R. A. (1999). Predictors of acute stress following motor vehicle accidents. Journal of Traumatic Stress, 12(3), 519-525. doi:10.1023/A:1024723205259

Holeva V., Tarrier N., Wells A. (2001). Prevalence and predictors of acute stress disorder and PTSD following road traffic accidents: Thought control strategies and social support. Behavior Therapy, 32, 65-83.

Lee S., Brasel K., Lee B. (2004). Emergency care practitioners' barrier to mental health assessment, treatment, and referral of post-injury patients. Wisconsin Medical Journal, 103(6), 78-82.

Lee S., Saunders S. (2004). Emergency care practitioners' attitudes and experiences regarding posttraumatic stress disorder. Journal of Trauma Practice, 3(1), 19-34.

National Institute for Clinical Excellence (NICE) (2005). The management of PTSD in adults and children in primary and secondary care. Wilshire, United Kingdom: Cromwell Press.

National Trauma Institute (NTI). (2014). Trauma statistics. Retrieved from

http://http://www.nationaltraumainstitute.org/home/trauma_statistics.html

Prins A., Ouimette P., Kimerling R., Cameron R. P., Hugelshofer D. S., Shaw-Hegwer L., Sheikh J. I. (2003). The primary care PTSD screen (PC-PTSD): development and operating characteristics. Primary Care Psychiatry, 9, 9-14.

Reese C., Pederson T., Avila S., Josephy K., Nagy K., Dennis A., Bokhari F. (2012). Screening for traumatic stress among survivors of urban trauma. Journal of Trauma and Acute Care Surgery, 73(2), 462-468.

doi:10.1097/TA.0b.13e31825ff713

Roy S. C. (2009). Elements of the Roy adaptation model. In The Roy Adaptation Model (3rd ed., pp. 29-62). Upper Saddle River, NJ: Pearson Education Inc.

Roy S. C., Andrews H. A. (1999). The Roy Adaptation Model (2nd ed.). Stamford, CN: Appleton \& Lange.

Spoont M., Arbisi P., Fu S., Greer N., Kehle-Forbes S., Meis L., Wilt T. J. (2013). Screening for post-traumatic stress disorder (PTSD) in primary care: A systematic review. Department of Veterans Affairs ESP Project. Retrieved from http://http://www.ncbi.nlm.nih.gov/books/NBK126691/pdf/Bookshelf_NBK126691.pdf 
Warren A. M., Stucky K., Sherman J. J., (2014). Rehabilitation psychology's role in the level I trauma center. Journal of Trauma Nursing, 21(3), 139-145.

World Health Organization (WHO). (2014). Violence and injury prevention. Retrieved from

http://http://www.who.int/violence_injury_prevention/media/news/2015/Injury_violence_facts_2014/en/

Zatzick D., Rivara F., Jurkovich G., Russo J., Trusz S. G., Wang J., Katon W. (2011). Enhancing the population impact of collaborative care interventions: Mixed method development and implementation of stepped care targeting posttraumatic stress disorder and related comorbidities after acute trauma. General Hospital Psychiatry, 33(2), 123-134.

Zatzick D. F., Rivara F. P., Nathens A. B., Jurkovich G. J., Wang J., Fan M. Y., Mackenzie E. J. (2007). A nationwide US study of post-traumatic stress after hospitalization for physical injury. Psychological Medicine, 37(10), 1469-1480. doi:10.1017/S0033291707000943 